\title{
Fabrication and properties of polyimide composites filled with zirconium tungsten phosphate of negative thermal expansion
}

\author{
XinWei Shi ${ }^{a}{ }^{*}$, Hong Lian ${ }^{a}$, XiaoSheng Yan ${ }^{a}$, Ruiqiong Qi ${ }^{a}$, Ning Yao ${ }^{a}$, Tao Li ${ }^{b}$ \\ ${ }^{\text {a }}$ School of Physical Science \& Engineering, Zhengzhou University, $100^{\text {th }}$ Science Road,
} Zhengzhou 450001, China

${ }^{\mathrm{b}}$ Department of Technology \& Physics, Zhengzhou University of Lightindustry, $5^{\text {th }}$ Dongfeng Road, Zhengzhou 450002, China

\begin{abstract}
Negative thermal expansion $\mathrm{Zr}_{2} \mathrm{WP}_{2} \mathrm{O}_{12}(\mathrm{ZWP})$ powder prepared by hydrothermal method was used as fillers to tailor the thermal expansion coefficient (TEC) of the polyimide (PI)-based composites. A series of PI-based composites containing different loading (0-40 wt\% or 0-19.6 vol\%) of ZWP powder were fabricated by the in-situ polymerization technique. Their structures and properties were characterized by Scanning electron microscope (SEM), Fourier transform infrared spectroscopy (FTIR), Impedance meter, Thermal mechanical analysis (TMA) and Thermogravimetric analysis (TGA). The additions of ZWP steadily reduced the TEC of the PI matrix at all loadings studied. A $40 \mathrm{wt} \%$ (19.6 vol\%) ZWP loading gives a 32.5\% (about $15 \times 10^{-6} / \mathrm{K}$ ) reduction of TEC. The thermal stability of the ZWP/PI composites can be enhanced with the increment of ZWP powder. The independence of the dielectric constant on frequency is improved by introduction of ZWP particles to PIs. The dielectric loss displays good stability, which indicates that the ZWP/PI composites
\end{abstract}

${ }^{*}$ Corresponding author. Tel.: +86 37167767832.

E-mail address: Shixw@zzu.edu.cn 
show potential applications in microelectronic and aerospace industries.

Keywords: thermal expansion; composite materials; dielectric properties; Fourier transform infrared spectroscopy (FTIR)

\section{Introduction}

Aromatic polyimides (PIs) are widely used in many engineering fields as buffer coatings, gas separation membrane, passivation layers, interlayer dielectrics, wafer-scale packages, etc., because of their desirable characteristics such as excellent mechanical properties, good thermal stability, chemical resistance, easy processability and low dielectric constant [1-6]. However, aromatic PIs exhibit relatively higher TEC (about $40-80 \times 10^{-6} / \mathrm{K}$ ) compared with most ceramics and metals (eg: $\mathrm{SiO}_{2}: 14$ $\times 10^{-6} / \mathrm{K}$, Si: $\left.3-5 \times 10^{-6} / \mathrm{K}, \mathrm{Cu}: 16-18 \times 10^{-6} / \mathrm{K}\right)$ [7]. The TEC value is a crucial factor for most structural and functional materials. A relatively higher TEC may cause serious destruction of devices [8], such as cracking or delamination. In the case of thin films, TEC is one of the key parameters that represent the degree of a film dimension changed during high temperature process [6]. Although PIs can be used in high temperature circumstances, the high TEC of which limits their use in a variety of advanced applications, such as solar panel used in spacecraft, conformal coatings used in the microelectronics industry and high performance structural materials in aerospace engineering [9, 10]. For example, solar panel is composed of several materials including cover glass, silicon rubber, silicon chip and PI, etc. The spacecraft experiences great temperature changes when working in orbit. Under such 
circumstances, the mismatch of TECs between PI and silicon rubber or silicon chip may lead to the reduction of spacecraft's reliability and service life. So, it is of great significance to improve the thermal expansion coefficient matching of PI with other materials. With the rapid development of electronic industry, the intent to improve the drawback derived from the TEC of PIs arouses more and more attentions.

In the plastics industry, the addition of filler materials to a polymer is a common and effective method to obtain a material with desired thermal properties [11]. To reduce the TEC of PIs, forming hybrid PI composites with inorganic filler materials is a promising approach [1]. In recent years, many researches have been carried out to tune the properties of PIs by incorporating inorganic materials, such as silica particles [1,12], $\mathrm{TiO}_{2}$ nanoparticles [13], montmorillonite [14], CNT [15], Ti [16] or $\mathrm{Al}_{2} \mathrm{O}_{3}$ [17], etc. However, the reduction in TEC by these particles is limited because of the positive TEC of the fillers [11].

Compared with most materials that exhibit positive TEC, Zirconium tungsten phosphate $\left(\mathrm{Zr}_{2} \mathrm{WP}_{2} \mathrm{O}_{12}\right.$, denoted as ZWP) displays negative thermal expansion (NTE) of approximately $-3 \sim-5 \times 10^{-6} / \mathrm{K}$ over a broad temperature range from room temperature to $1073 \mathrm{~K}$ [18-20]. ZWP comprises $\mathrm{ZrO}_{6}$ octahedra sharing corners with $\mathrm{WO}_{4}$ and $\mathrm{PO}_{4}$ tetrahedra [21] and remains orthorhombic structure down to very low temperatures $[18,22]$. The NTE behavior of this material results mainly from transverse motions of the bridging $\mathrm{O}$ atoms, or the coupled rotation between the octahedral and tetrahedral [23]. Compared with other NET materials reported [24], ZWP is stable under certain pressure and over a large temperature range $[25,26]$. 
ZWP also has desirable bending strength and low dielectric constants. When ZWP is used as filler in composites, no phase transition will occur. From an economic perspective, ZWP is a low-cost raw material. Isobe, et al. pointed out that ZWP is a novel NTE material [26]. Hence, ZWP should be an excellent and promising candidate for use as an inorganic filler to control the TECs of ceramics, metals, polymers and glasses. However, there are very few reports about ZWP used as filler to tune the TECs of other materials, especially to tune the TECs of PI.

The objective of this presentation is to develop PI-based composites with reduced TEC and also to maintain other favorable bulk properties of the polymer, such as thermal stability and dielectric properties. Thus, ZWP/PI hybrids with different ZWP loading were prepared and the properties of which were investigated.

\section{Experimental}

\subsection{Materials}

$\mathrm{ZrOCl}_{2} \bullet 8 \mathrm{H}_{2} \mathrm{O}, \mathrm{H}_{42} \mathrm{~N}_{10} \mathrm{O}_{42} \mathrm{~W}_{12} \bullet \mathrm{xH}_{2} \mathrm{O}$ and $\left(\mathrm{NH}_{4}\right) \mathrm{H}_{2} \mathrm{PO}_{4}$ with $99 \%$ purity were purchased from ALADDIN REAGENT (Shanghai) co., Ltd. N,N-dimethylformamide (DMF) was purchased from fine chemical engineering research and development Center of GuangDong province, China. Pyromellitic dianhydride(PMDA) and 4, 4' -oxydianiline (ODA) were purchased from Chinese Chemical Reagent Co. Ltd. And other reactants and solvents were used as received if it is not mentioned specifically.

\subsection{Sample preparation}

Hydrothermal method was used to prepare $\mathrm{ZWP}$ powder. $\mathrm{ZrOCl}_{2} \bullet 8 \mathrm{H}_{2} \mathrm{O}$, $\mathrm{H}_{42} \mathrm{~N}_{10} \mathrm{O}_{42} \mathrm{~W}_{12} \bullet \mathrm{xH}_{2} \mathrm{O}$ and $\left(\mathrm{NH}_{4}\right) \mathrm{H}_{2} \mathrm{PO}_{4}$ with the molar ratio of $\mathrm{Zr}: \mathrm{W}: \mathrm{P}=2: 1: 2$ were 
maintained. During the preparation process, $\left(\mathrm{NH}_{4}\right) \mathrm{H}_{2} \mathrm{PO}_{4}$ aqueous solution was added into $\mathrm{H}_{42} \mathrm{~N}_{10} \mathrm{O}_{42} \mathrm{~W}_{12} \bullet \mathrm{xH}_{2} \mathrm{O}$ aqueous solution. After reaction, a transparent solution will be formed. And then $\mathrm{ZrOCl}_{2} \cdot 8 \mathrm{H}_{2} \mathrm{O}$ was dropwise added into the obtained transparent solution under magnetic stirring and a slurry was formed rapidly. The obtained slurry (ZWP precursor) was then put into a sealed reaction kettle (HZSF12-type, made by GONG YI INSTRUMENT AND EQUIPMENT CO. , LTD., Henan province, China.). After reacting for $48 \mathrm{~h}$ at $130^{\circ} \mathrm{C}$, the reaction mixture was then taken out and cleaned in a centrifuge for 5 times to obtain the precipitates. The precipitates were filtrated and dried at $80^{\circ} \mathrm{C}$ in a vacuum drying oven, followed by annealing in a muffle furnace at $900^{\circ} \mathrm{C}$ for $4 \mathrm{~h}$ to get white ZWP powder. Usually, hydrothermal methods can yield nanoparticles. However, the ZWP powder we prepared was a little rough. So, the obtained powder was then ball mixed in a planetary muller for $10 \mathrm{~h}$ to refine the particles. Furthermore, thinner particles may improve the composite effects [27]. After fully ball milling, the powder was pressed by cold pressing to form $\Phi 10 \mathrm{~mm} \times 4 \mathrm{~mm}$ cylinders. The obtained cylinders were sintered at $900^{\circ} \mathrm{C}$ for $4 \mathrm{~h}$ and cooled along with the furnace for thermal expansion coefficient test [28].

ZWP/PI hybrid composites containing different loading ZWP powder were fabricated by in-situ polymerization technique. A $1 \mathrm{~g}$ of the vacuum dried diamine monomer (ODA) was added into $20 \mathrm{~mL}$ DMF. The mixture was stirred thoroughly at room temperature to obtain a clear solution. Different contents $(0 \mathrm{wt} \%, 5$ wt \%, 20 wt $\%, 30$ wt $\%, 40$ wt $\%$ or 0 vol $\%, 1.9$ vol $\%, 8.4$ vol\%, 13.6 vol $\%, 19.6$ vol\%) of ZWP powder were then added into this solution and allowed to mix by stirring until 
the solution became white. A $1.145 \mathrm{~g}$ of the vacuum dried dianhydride monomer (PMDA) was added gradually during 40-60min. And at the same time, the combined reaction mixture was stirred at room temperature for $4-5 \mathrm{~h}$ by a magnetic stirrer. A mixed solution of PAA/ZWP/DMF (PAA is the abbreviation of polyamic acid, the precursor of PI) with pale-yellow color was obtained. The mixed solution was dried at $80^{\circ} \mathrm{C}$ for $24 \mathrm{~h}$, followed by heat-treating for $1 \mathrm{~h}$ at 120 and $180^{\circ} \mathrm{C}$, and then at 250 and $300^{\circ} \mathrm{C}$ for $4 \mathrm{~h}$ with temperature increasing rate of $5^{\circ} \mathrm{C} / \mathrm{min}$ [8]. After the above mentioned heat treatment, a complete imidization of PAA to PI would be obtained. The as-prepared ZWP/PI blended powder was grinded and pressed into cylinders of $\Phi 10 \mathrm{~mm} \times 4 \mathrm{~mm}$ for the following characteristic measurements.

\subsection{Instrumentation and measurements}

X-ray diffraction (XRD) analysis of the as-prepared ZWP powder was carried out with the BRUKER D8 Advance Diffractometer (German) using a $\mathrm{Cu} \mathrm{K}_{\alpha}$ X-ray source $(\lambda=0.15406 \mathrm{~nm}$ ) operating at $40 \mathrm{KV}$ and $740 \mathrm{~mA}$, scanning at a rate of $2 \% \mathrm{~min}$ in the $2 \theta$ range from 10 to 33 degrees. Scanning electron microscopic (SEM) micrographs were obtained on a JSM-6700F/INCA-ENERGY (Japan) SEM. Energy dispersive X-ray analysis (EDX) studies were recorded on a field emission scanning electron microscope (Nova 400 nanoSEM, FEI Company, USA) equipped with an energy dispersive X-ray spectroscope (INCA IE 350 PentaFET X-3,Oxford, UK). The particle size of the as-prepared ZWP powder was measured through SEM image by Nano Measurer 1.2 program [29] and confirmed by Zetasizer Nano ZS90 (MALVERN, England). Thermal mechanical analysis (TMA) of the as-prepared ZWP 
cylinder and ZWP/PI composites were obtained on a LINSEIS-L76 (German). The relative densities of the as-prepared ZWP ceramic and ZWP/PI composites were calculated by using Archimedes technique. The ZWP/PI composites were analyzed by Fourier Transform Infrared (FTIR) spectrometer. FTIR spectra were acquired on a Nicolet8700 (USA) in the wavenumbers of $500 \sim 4000 \mathrm{~cm}^{-1}$. The dielectric properties of the ZWP/PI hybrid composites were acquired from AGILENT4294A Impedance meter (USA). The thermogravimetric analysis curve of the ZWP/PI composites was recorded on a LABSYS ${ }^{\mathrm{TM}}$ thermogravimeter (SETARAM, French).

\section{Results and discussion}

\subsection{Characterization and Thermal expansion coefficient of ZWP}

Fig. 1 portrays the XRD pattern of the as-prepared ZWP powder. It can be seen from the figure that the diffraction peaks are high and sharp which implies high crystallinity of ZWP powder. Each of the diffraction peaks in Fig. 1 can be exactly indexed to ZWP (PDF No. 00-023-0755). No other miscellaneous peaks (such as $\mathrm{ZrO}_{2}, \mathrm{WO}_{3}$, etc.) were observed, which indicates that pure and monophase ZWP has been obtained via hydrothermal method by heating at $900^{\circ} \mathrm{C}$ for $4 \mathrm{~h}$.

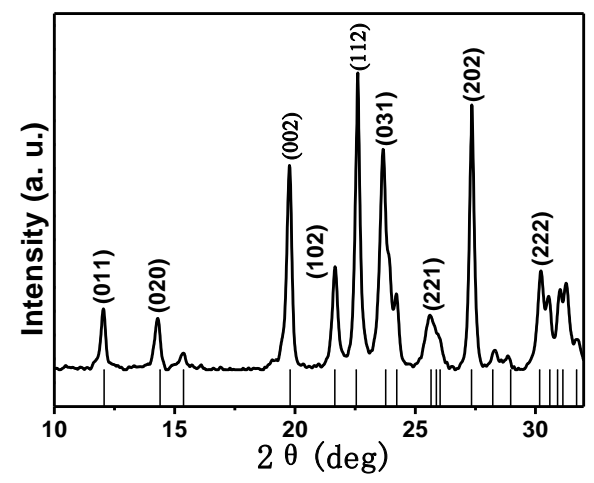

Fig. 1 XRD pattern of ZWP powder

Fig. 2 illustrates SEM image and the particle size distribution of the obtained 
ZWP powder. It is evident that the obtained powder exhibits a rugged shape with some pores. The particle size of the ZWP powder was around 320nm (Fig.2 b) measured by nano measurer 1.2 program through SEM image [29], at least 100 particles were selected for analysis. The particle size was also tested by Zetasizer Nano ZS90 and illustrated in the inset of Fig.2 b. The two values are in good agreement. Compared with the particle sizes reported in the literatures $[8,26]$, the ZWP powder prepared by hydrothermal method is thinner and finer. This may be attributed to the ball milling and the chemical reaction during the hydrothermal method. Hydrothermal technology is a kind of wet chemical method. In our previous study, the chemical reaction of ZWP by hydrothermal method was inferred from the perspective of ion coexist [28]. This method presents many benefits such as use of mild temperature and pressures, elimination of high-temperature calcinations and removing aggregates $[30,31]$. Thus, powders prepared by this method are thin and fine. It is presumed that fillers added to polymers with small size, such as ultrafine or nanoparticles, can improve the properties of the composites [27]. Cora Lind and Sharma et al. reported that NTE fillers of thin or nano-sized particles will be beneficial to the composite, Such as reducing agglomeration, improving the affinity between NTE fillers and matrices, and so forth [7, 32, 33]. So, it is of interest to study the effect of particle size on the bulk properties of polymers including thermal expansion [7]. Thorough research of the influences of particle size and structure on the properties of ZWP may help to widen the doors of this new material used in engineering field. Detailed research will be carried out to understand the effect of the particle size on the 
property of ZWP ceramics or its composites in the very future.
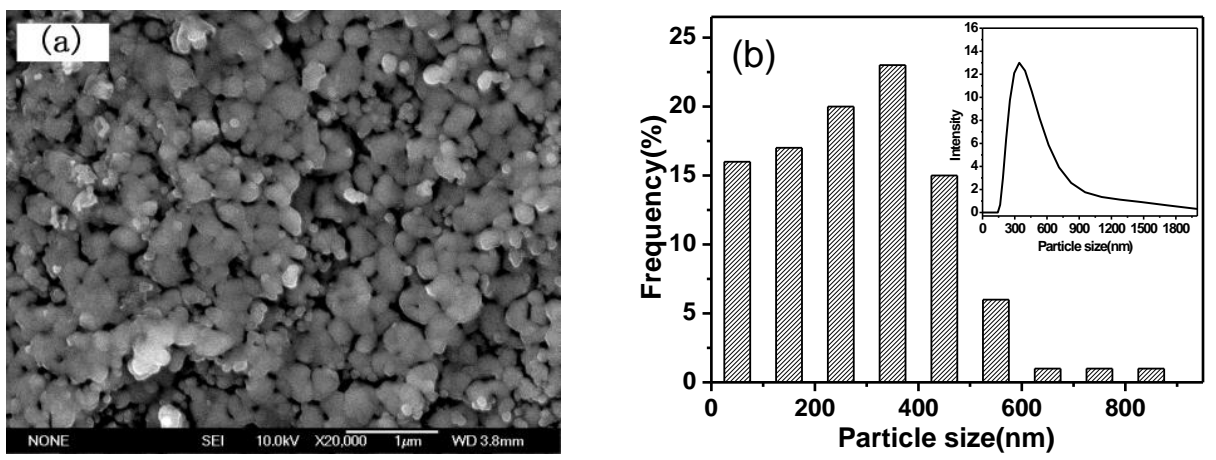

Fig. 2 SEM image of the obtained ZWP powder (a) and particle size distribution (b)

The coefficient of thermal expansion $(\alpha)$ of a material at constant pressure can be calculated according to Eq (1) [34]:

$$
a=\frac{1}{L_{0}} \cdot \frac{d_{L}}{d_{T}}
$$

Where $d_{L} / d_{T}$ is the change rate of the sample length with temperature, and $L_{0}$ is the initial sample length. Fig. 3 presents the relative length changes $\left(d L / L_{0}\right)$ of the ZWP ceramics. As can be seen, the ZWP ceramics shrank with increasing temperature. According to $\mathrm{Eq}$ (1), the mean linear coefficient was calculated as about $-2.012 \times 10^{-6} / \mathrm{K}$. Evans et al. reported that mean linear coefficients of ZWP estimated by dilatometer method and XRD measurement were -6 and $-3 \times 10^{-6} / \mathrm{K}$, respectively[19]. Isobe et al. reported that the TEC of ZWP ceramics made by solid-state method was $-3.4 \times 10^{-6} / \mathrm{K}$, which was measured by a thermomechanical analyzer from 30 to $600^{\circ} \mathrm{C}$ [26]. So, the TEC of the present study is a little lower than those reported. The probable reason is the difficult densification of ZWP ceramics pressed by cold pressing method and sintered with no sintering additive [26]. The relative density of the ZWP ceramic calculated by using Archimedes technique was 
around 53\%, which is a little lower than that of Isobe reported (about 60\%) [26]. In this case, the porosity in the material would expand when ZWP ceramic was heated during the TMA test. The expansion force of the porosity may weaken the contraction of ZWP ceramic. This may be the reason why the present TEC value is a little lower than that of the literature reported [26]. From Fig. 3, we can also find that the curve is smooth and there is no steep slope, which illustrates that no phase transition occurs in ZWP ceramics over the measured temperature range. This is the reason why ZWP is an excellent candidate of inorganic filler to tailor the TEC of PIs.

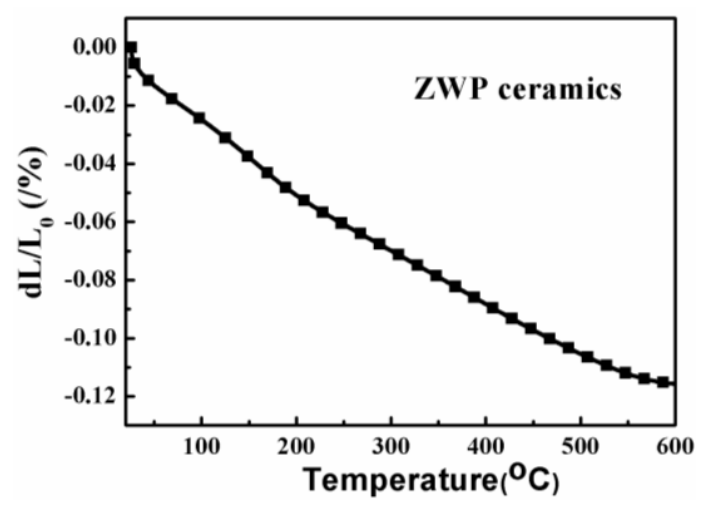

Fig. 3 Relative length change with temperature (on heating) of ZWP ceramic

\subsection{Fourier Transform Infrared spectra of ZWP/PI composites}

The obtained ZWP powder was used as fillers in ZWP/PI to control the TEC of the composites. To characterize the chemical bonding nature of the obtained ZWP/PI composites and PI, the typical transmission FTIR spectra is shown in Fig. 4. It can be seen from the figure that there are obvious peaks at $3433 \mathrm{~cm}^{-1}, 1720 \mathrm{~cm}^{-1}, 1380 \mathrm{~cm}^{-1}$ and $729 \mathrm{~cm}^{-1}$, respectively, which indicates a complete imidization in PI and ZWP/PI hybrids. The peak at about $3400 \mathrm{~cm}^{-1}$ is generated from the hydroxyl and amino groups [35]. The existence of these polar groups of hydroxyl and amino will cause the 
water absorption, which will bring down the thermal stability of the composite. According to the date reported in the document (Table 1) [36], the peaks at about $1780 \mathrm{~cm}^{-1}(L \mathrm{C}=\mathrm{O}$, amide band I peak $)$ and $1720 \mathrm{~cm}^{-1}(\mathscr{L}=\mathrm{O}$, amide band II peak $)$ are the typical asymmetric and symmetric stretching, respectively. Other peaks originating from vibrational modes of the imide ring are located at $1380 \mathrm{~cm}^{-1}$ $\left[\nu(\mathrm{C}-\mathrm{N})(\mathrm{O}-\mathrm{C})_{2}(\mathrm{~N}-\mathrm{C})\right.$, amide band III peak $]$ and $725 \mathrm{~cm}^{-1}(\nu \mathrm{C}=\mathrm{O}$, in-plane imide ring deformation, imide band IV peak) [37]. As reported, in highly imidized PIs, the peaks at $1780 \mathrm{~cm}^{-1}$ and $725 \mathrm{~cm}^{-1}$ are weak and insensitive [36], so the $1380 \mathrm{~cm}^{-1}$ peak can be selected to indentify the imidization. The peak at around $1069 \mathrm{~cm}^{-1}$ also indicated the complete conversion of PAA to PI [4]. The results from the FTIR spectra are in good agreement with those reported in the above literatures $[4,36,37]$. The absence of the band at about $1665 \mathrm{~cm}^{-1}$ further confirmed a complete imidized structure of PI and ZWP/PI hybrids [8]. It is worth mentioning that there is a change at $1031 \mathrm{~cm}^{-1}$ and $861 \mathrm{~cm}^{-1}$ for ZWP/PI. This may be due to the characteristic stretching vibrations of $\mathrm{W}=\mathrm{O}$ bond $[38,39]$ for the reason of ZWP incorporating in PI. The FTIR spectra of Fig.4 (a) and (b) have no other differences, which indicate the adding of ZWP powder has no effect on the PI structure.

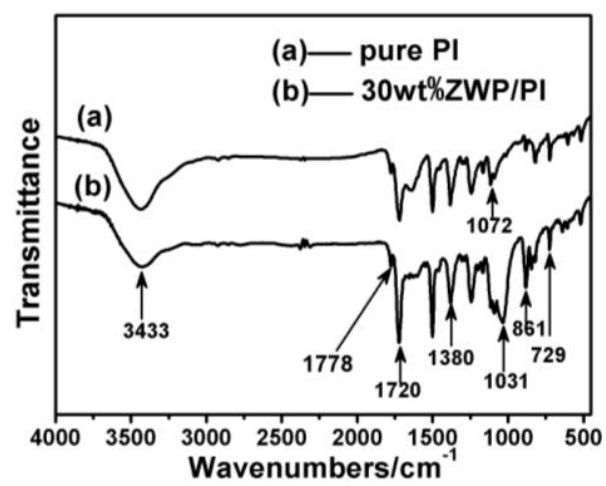

Fig. 4 FTIR spectra of (a) pure PI and (b) $30 \mathrm{wt} \%$ ZWP/PI hybrid composite 
Table 1 FTIR spectra of PI or its hybrids [36]

\begin{tabular}{cccc}
\hline & Absorption band $/ \mathrm{cm}^{-1}$ & Intensity & Vibrational type \\
\cline { 2 - 4 } Aromatic PIs or & 1780 & $\mathrm{~S}$ & $\mathrm{C}=\mathrm{O}$ asymmetric stretching \\
hybrids & 1720 & $\mathrm{VS}$ & $\mathrm{C}=\mathrm{O}$ symmetric stretching \\
& 1380 & $\mathrm{~S}$ & $\mathrm{C}-\mathrm{N}$ stretching \\
& 725 & $\mathrm{~W}$ & $\mathrm{C}-\mathrm{O}$ deformation \\
\hline
\end{tabular}

Notes

S: Strong, VS: Very strong, W: Weak

3.3 Scanning electron microscopic image of ZWP/PI composites

Fig. 5 portrays the typical SEM micrograph of the top surface and fracture surface of $30 \mathrm{wt} \%$ ZWP/PI hybrid composite, respectively. The top surface photograph indicates that some ZWP powder dispersed in the PI matrix in an exfoliation morphology, while more powder is embedded in the PI matrix. These embedded particles with small size may be benefit to the improvement of thermal properties [12].

In order to observe the inner structure of the obtained ZWP/PI hybrid composites, the samples were quenched in liquid nitrogen and brittle fractured. The fracture surface graph is given in Fig.5 (b). It can be found that the ZWP powder scattered homogeneously in the colloidal PI matrix in the form of crosslinking. This phenomena may have an impact on the performances (eg: thermal stability, dielectric properties, etc) of the composites. However, it is obvious that in the inside of the composites, the two phases of the ZWP particles and PI matrix are isolated by pores and cracks, which indicates the preparation process of ZWP/PI hybrid composites should be further improved. These defects between the fillers and the PI matrix may be reduced or even eliminated by adding coupling agents, such as ( $\gamma$-glycidyloxypropyl)trimethoxylane [37] or aminophenyl-trimethoxysilane [40] . Concerning the chemical composition of the composites, energy dispersive X-ray 
(EDX) analysis (Fig.5 c) revealed the presence of zirconium ( $\mathrm{Zr}$ ), tungsten (W), phosphorus (P) and oxygen (O). The results are concordant with that of XRD (Fig.1).
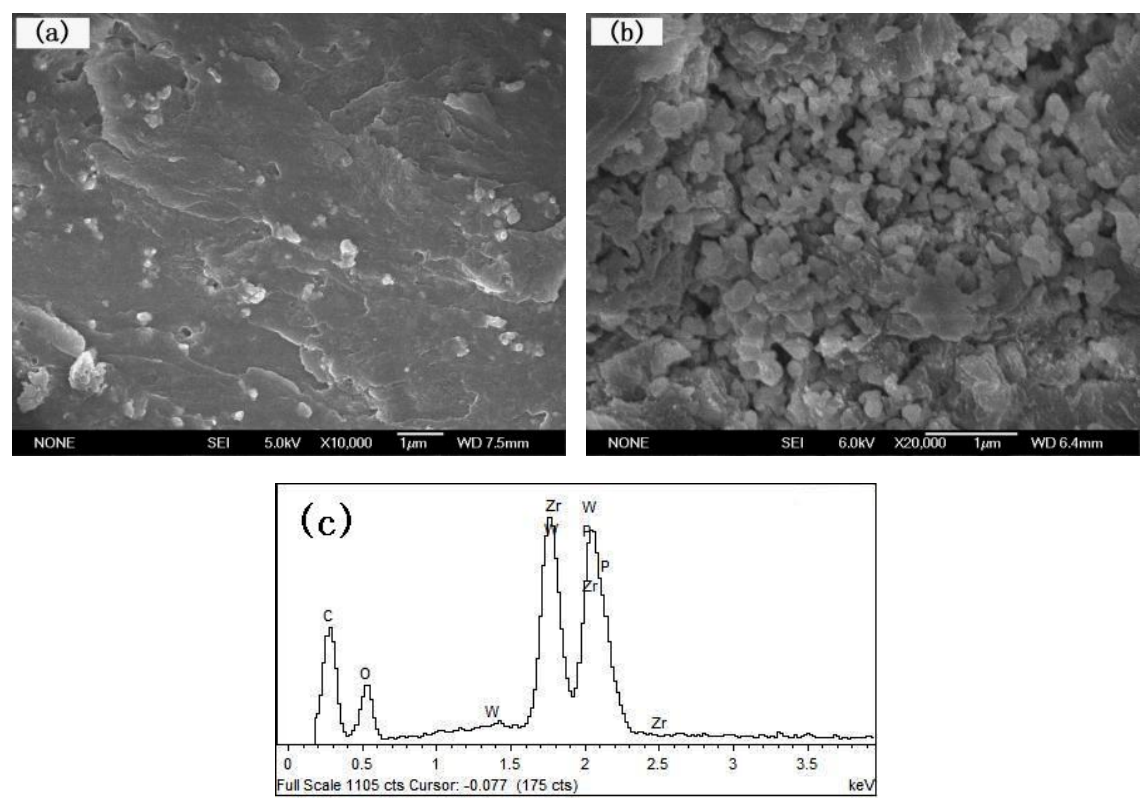

Fig. 5 SEM micrograph of $30 \mathrm{wt} \%$ ZWP/PI hybrid (a) top surface, (b) fracture surface, (c) EDX

patterns

\subsection{Thermal expansion coefficients of ZWP/PI composites}

TECs of the as-prepared ZWP/PI composites were measured using a TMA and calculated according to Eq (1). Fig.6 shows the relationship between the ZWP powder loading and the average TEC values of the ZWP/PI composites (including ZWP ceramic) from room temperature to $350^{\circ} \mathrm{C}$. It can be seen from the figure that introduction of ZWP powder would lead to a steady decrease in the TEC of the ZWP/PI hybrids. With the increasing of ZWP powder loading, the TEC decreased from $45.67 \times 10^{-6} / \mathrm{K}$ for the pristine PI to $30.81 \times 10^{-6} / \mathrm{K}$ for the ZWP/PI hybrid with 40 wt\% (19.6 vol\%) ZWP loading (inset in Fig.6). The density of porosity of pure PI and the obtained ZWP/PI composites is given in Table 2. It can be seen that with the increment of ZWP, the density of porosity increased. In this case, the porosity may 
accommodate the expansion of the positive thermal expansion material when heated during the TMA test. Hence, TEC of the positive thermal expansion material may increase. However, the TEC of the obtained ZWP/PI composites reduced with increment of ZWP in the composites (Fig.6). From this point of view, the effect of ZWP in reducing the TEC of ZWP/PI composite is prominent.

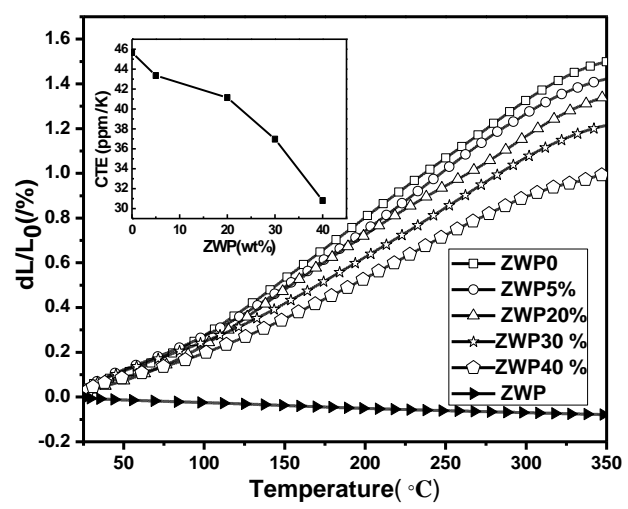

Fig. 6 Relationship between the ZWP powder loading and the TECs of the ZWP/PI composites

Table 2 The density of porosity of pure PI and the obtained ZWP/PI composites

\begin{tabular}{cccccc}
\hline ZWP content(wt $\%)$ & Pure PI & $5 \%$ ZWP/PI & $20 \%$ ZWP/PI & $30 \%$ ZWP/PI & $40 \%$ ZWP/PI \\
density of porosity & $16.5 \%$ & $16.6 \%$ & $16.8 \%$ & $17.5 \%$ & $20.0 \%$ \\
\hline
\end{tabular}

The Rule of Mixtures (ROM) model, which does not account for any type of interactions that may exist between the polymer and the filler, serves as the first-order approximation to calculate the TEC of the composites $[7,11]$.

$$
\alpha_{c}=\alpha_{\mathrm{f}} \Phi+\alpha_{\mathrm{m}}(1-\Phi)
$$

Where $\alpha_{c}, \alpha_{\mathrm{f}}$, and $\alpha_{\mathrm{m}}$ represent the TEC value of the composite, the matrix and the filler, respectively. $\Phi$ is the volume fraction of the filler.

The experimental and the calculated values by ROM model are illustrated in Fig.7. The experimental values are lower than those calculated. From this point of view, ROM doesn't take into account any type of defects or the interaction between the 
filler and the PI matrix, so the calculated values are higher than those of experimental. In fact, the ZWP powder interacts with PI matrix, as deduced from the SEM analysis (Fig.5). An addition of $40 \mathrm{wt} \%$ (19.6 vol\%) ZWP can reduce the TEC of the pristine PI by around $32.5 \%$ (about $15 \times 10^{-6} / \mathrm{K}$ ). Therefore, the introduction of NTE ZWP powder to PI is effective in reducing the TECs of ZWP/PI hybrids. However, compared with the TEC values of $\mathrm{Si}\left(3-5 \times 10^{-6} / \mathrm{K}\right), \mathrm{Cu}\left(16-18 \times 10^{-6} / \mathrm{K}\right)$ and $\mathrm{SiO}_{2}$ $\left(14 \times 10^{-6} / \mathrm{K}\right)$, the decrease in TEC is extremely desired when PI is used in the microelectronic and aerospace industries. Further work needs to be carried out to reduce the TECs of PIs more prominent.

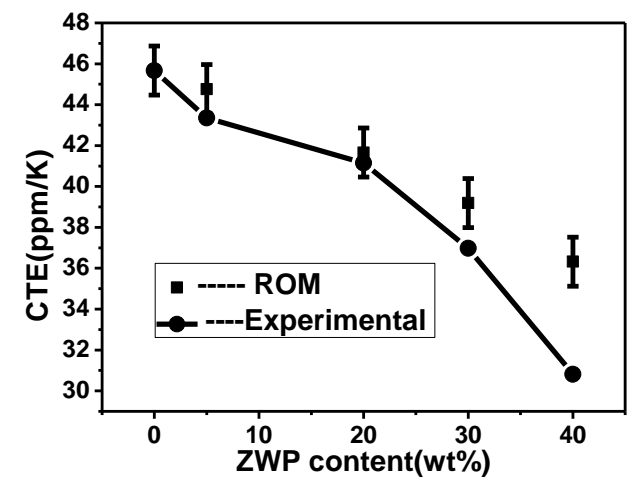

Fig. 7 TEC values of experiment and calculated by ROM model of ZWP/PI hybrids with different

ZWP powder loading

3.5 Thermogravimetric analysis of ZWP/PI composites

Fig. 8 shows the thermogravimetric analysis (TGA) curves of ZWP/PI composites with different ZWP loading, recorded in air at $10^{\circ} \mathrm{C} / \mathrm{min}$. It can be seen from the figure that the thermal stability increases with ZWP loading. There is a little weight loss before $450^{\circ} \mathrm{C}$ (Fig.8.a). This may be attributed to the absorbed waters (as indicated in FTIR spectra, peak at $3433 \mathrm{~cm}^{-1}$ ). But for the pure PI, the degradation rate became fast beyond that temperature, while the ZWP/PI hybrid composites with 
different content of ZWP powder decomposed slowly. This trend kept until high temperature of around $570^{\circ} \mathrm{C}$. Evidently, the introduction of ZWP fillers remarkably improves the thermal stability, shifting the TGA curves to higher temperatures.

Fig. 8(b) shows the decomposition temperatures at which $10 \mathrm{wt} \%$ weight loss $\left(\mathrm{T}_{10}\right)$ occurred for the ZWP/PI hybrid composite. The $\mathrm{T}_{10}$ was enhanced about $83^{\circ} \mathrm{C}$, which can be obtained from the $\mathrm{T}_{10}$ of the pure PI $\left(527^{\circ} \mathrm{C}\right)$ and the ZWP/PI composite with 40 wt $\%$ ZWP loading $\left(610^{\circ} \mathrm{C}\right)$. The values of $\mathrm{T}_{10}$ increase with increasing ZWP loading, which indicates the thermal stability of pure PI can be enhanced by incorporating ZWP fillers. This trend may be attributed to the complete imidization of PI and uniform dispersion of ZWP particles in colloidal PI matrix. From the results of FTIR (Fig.4) and SEM (Fig.5), ZWP particles (about 320nm in size) disperse in fully imidized PI matrix. These small particles may have strong interaction with the colloidal PI matrix and can act as efficient physical cross-linking points to restrict the segmental motion of polymers $[12,29]$. With the increment of ZWP powder, more powder acting as the efficient physical cross-linking points would be obtained. Hence the thermal stability of the ZWP/PI was enhanced with the ZWP loading.
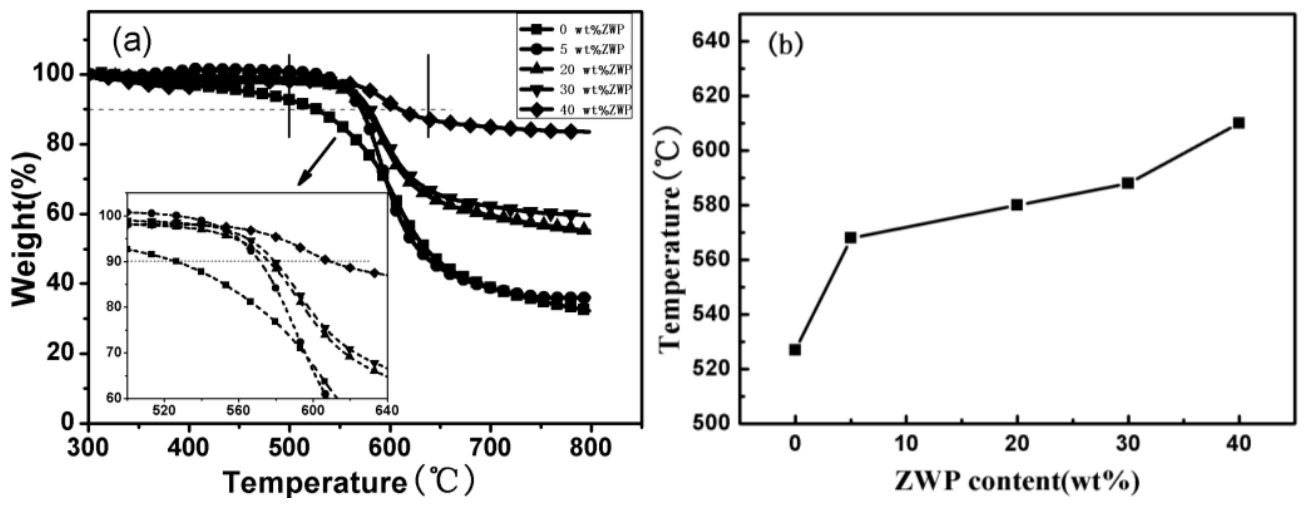

Fig. 8 TG curves of ZWP/PI hybrids with different ZWP powder loading 


\subsection{Dielectric properties of ZWP/PI composites}

It is well known that the dielectric property is another crucial parameter when PIs are used in microelectronic and aerospace fields. However, the dielectric property is significantly affected by many factors, such as, the molecular packing, chain order, morphological structure, crystallinity and composition of the materials [3]. Therefore, the dielectric property of the ZWP/PI hybrids was also investigated.

The dielectric behavior of a dielectric material under the action of an alternating charging current is often described by a frequency-dependent complex dielectric constant, $\varepsilon(j \omega)$ and can be defined as follows[41]:

$$
\varepsilon(j \omega)=\varepsilon^{\prime}(j \omega)-\varepsilon^{\prime \prime}(j \omega)
$$

Where $\varepsilon^{\prime}(\omega)$ and $\varepsilon^{\prime \prime}(\omega)$ are the dielectric constant and the dielectric loss $(\tan \delta)$, respectively. The Dielectric constant is the ability of a dielectric material to store electric potential energy under the influence of an electric field and can be calculated by Eq (4) [42]:

$$
\varepsilon^{\prime}=\mathrm{C}_{\mathrm{P}} \cdot \mathrm{t} / \varepsilon_{0} \cdot \mathrm{S}
$$

Where $C_{P}$ is the measured value of the capacitance, $t$ is the average thickness of the sample, $\varepsilon_{0}$ is permittivity of vacuum $\left(8.85 \times 10^{-12} \mathrm{farad} / \mathrm{m}\right)$, and $\mathrm{S}$ is the surface area of the sample. The loss tangent is a parameter that measures the inherent energy dissipation (loss) of electromagnetic field of a dielectric material and can be defined as the ratio of the loss current to the charging current in the sample. For PIs used in microelectronic and aerospace fields as interlayer dielectrics, the lower the loss tangent is, the better they will service the applications. 


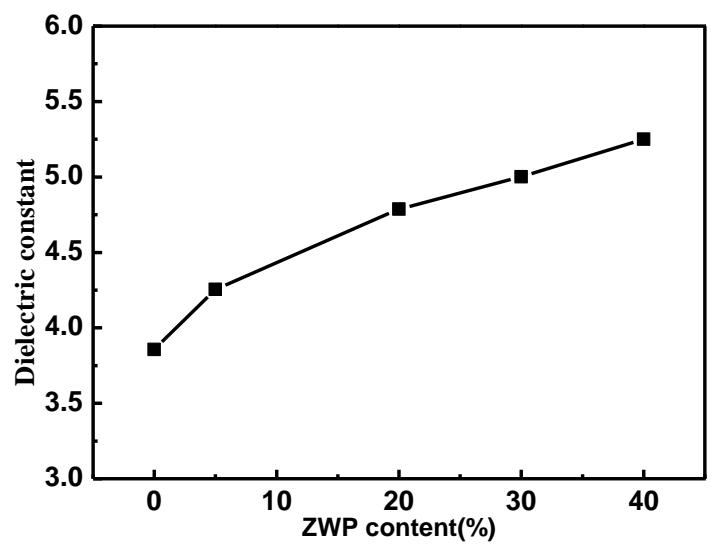

Fig.9 Dielectric constants of the obtained ZWP/PI hybrids with different ZWP powder loading in a

$10 \mathrm{KHz}$ field at room temperature

Fig.9 shows the dependence of the dielectric constants of the resulting ZWP/PI composites in a $10 \mathrm{KHz}$ field at room temperature calculated by Eq (4). As can be seen, the dielectric constant of the ZWP/PI composites increased with an increase of the ZWP powder loading. The dielectric constant of the pure PI and ZWP/PI with 40 wt $\%$ ZWP is 3.856 and 5.241, respectively. The reason for the increase of dielectric constant with ZWP loading can be due to the inherently higher dielectric constant of ZWP relative to that of the PI and the small ZWP particles dispersed in the hybrid composites (as shown in SEM image, Fig.5.b). Even though, the exact dielectric constant of ZWP ceramics is not easy to measure. Its dielectric constant is higher than that of Pure PI (date will be reported later). Naturally, as ZWP content increases in the composites, their dielectric constant increases. On the other hand, the small ZWP particles disperse in the PI matrix (Fig.5) and have a large interface charge polarization, which will lead to a relative increase in dielectric constant [43]. This kind of phenomenon appears in the heterogeneous system like polymer-ceramic composite, as T. Badapanda et al. pointed out [44], due to the accumulation of the 
electric charges at the boundaries and the formation of the large dipoles on the ceramic particles or clusters. With the increment of ZWP content in the hybrid composites, the degree of interface polarization becomes more prominent. As a result, the dielectric constant increases monotonically with increasing of ZWP content. The dielectric constant values of present study are higher than those reported in literatures. Further work needs to be done to reduce the dielectric constant of ZWP/PI composites.

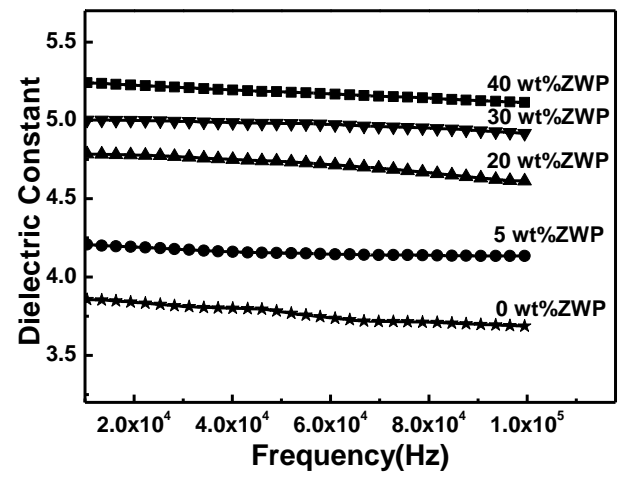

Fig.10 Variation of dielectric constants with frequency of the ZWP/PI composites at room temperature from $10 \mathrm{KHz}$ to $100 \mathrm{KHz}$

Fig. 10 illustrates the variation of $\varepsilon^{\prime}$ with frequency of the ZWP/PI composites at room temperature from $10 \mathrm{KHz}$ to $100 \mathrm{KHz}$. It can be found that with the increasing of the frequency, the dielectric constants of the resulting ZWP/PI composites all have a slight decrease. This may be due to the dielectric relaxation of the ZWP ceramics and the interfaces between PI matrix and ZWP powder in the composites [44]. However, the decreasing amplitude is very weak. Table 3 gives the decreasing amplitude of the composites' dielectric constant with different ZWP loading at low and high frequency, respectively. It can be seen that all the decreasing amplitude of the composites with different ZWP loading(5-40 wt\%) is lower than that of pure PI, which indicates that 
the independence of the dielectric constant on frequency is improved by adding ZWP to PIs.

Table 3 The decreasing amplitude of $\varepsilon^{\prime}$ with different ZWP loading at low and high frequency

\begin{tabular}{cccc}
\hline ZWP content $(\mathrm{wt} \%)$ & $\varepsilon^{\prime}$ at $10 \mathrm{KHz}$ & $\varepsilon^{\prime}$ at $100 \mathrm{KHz}$ & decreasing amplitude(\%) \\
\hline 0 & 3.856 & 3.687 & 4.383 \\
5 & 4.208 & 4.135 & 1.735 \\
20 & 4.785 & 4.613 & 3.595 \\
30 & 5.002 & 4.917 & 1.699 \\
40 & 5.241 & 5.114 & 2.423 \\
\hline
\end{tabular}

Fig.11 shows the dependence of the dielectric loss of the resulting ZWP/PI hybrids in a $10 \mathrm{KHz}$ field at room temperature calculated by eq (4). It can be seen that the loss tangent of the resulting composites was all below 0.004. As ZWP content increased from $0 \mathrm{wt} \%$ to $40 \mathrm{wt} \%$, the loss tangent slightly increased from 0.00382 to 0.00398. The increased percentage is only $4.18 \%$. These results reveal that with the ZWP content increasing, the dielectric loss tangent retained relative low and stable nature. The low and stable loss tangent is desirable for PIs used in microelectronic and aerospace fields. Both the weak dependence of the dielectric constant on frequency and the low and stable loss tangent are desirable for future dielectric materials [45].

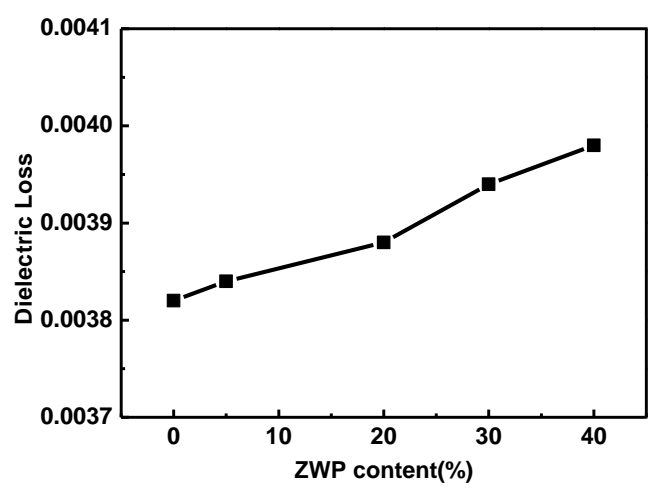

Fig.11 dielectric loss of the obtained ZWP/PI hybrids with different ZWP powder loading in a $10 \mathrm{KHz}$ field at room temperature 


\section{Conclusions}

In this study, $\mathrm{Zr}_{2} \mathrm{WP}_{2} \mathrm{O}_{12}$ /polyimide hybrid composites were successfully prepared by in-situ polymerization technique using the self-prepared negative thermal expansion $\mathrm{Zr}_{2} \mathrm{WP}_{2} \mathrm{O}_{12}$ as fillers. The $\mathrm{ZWP}$ particles were well-dispersed in the colloidal PI matrix in a form of cross-linking as observed from SEM image. The TECs of the ZWP/PI composites decrease with the increasing of ZWP powder. A 40 wt $\%\left(19.6\right.$ vol\%) ZWP loading can give a $32.5 \%$ (about $\left.15 \times 10^{-6} / \mathrm{K}\right)$ reduction of TEC. The thermal stability of the composites was also enhanced by incorporating ZWP particles. The dielectric study revealed that the dielectric constant $\left(\varepsilon^{\prime}\right)$ and the dielectric loss of the composites increase with the increase of the ZWP loading. The independence of the dielectric constant on frequency is improved by adding ZWP to PIs. The dielectric loss values are all below 0.004 at all loadings of ZWP particles. Low loss tangent and stable dielectric properties might make the ZWP/PI hybrid composites as a good candidate material in microelectronic and aerospace industries.

\section{Acknowledgments}

This work was supported by the National Science Foundation of China (No. 10974183). We are grateful to Professor Zhenping Chen of Department of Technology and Physics, Zhengzhou University of LightIndustry, China for the measurements of dielectric properties. The authors would also be grateful to Professor Mingju Chao of the Key Laboratory of Materials Physics of Ministry of Education of China, Zhengzhou University for the measurements of TECs of the samples. 


\section{References}

[1] Seong-Ku Kim, Xingyuan Wang, Shinji Ando, Xiaogong Wang, Highly transparent triethoxysilane-terminated copolyimide and its $\mathrm{SiO}_{2}$ composite with enchanced thermal stability and reduced thermal expansion, Eur. Polym. J. 64 (2015) 206-214.

[2] Jun-chao Huang, Chao-bin He, Yang Xiao, Khine Yi Mya, Jie Dai, Yeen Ping Siow, Polyimide/POSS nanocomposites: interfacial interaction, thermal properties and mechanical properties, Polym. 44 (2003) 4491-4499.

[3] J. C. Tang, H. C. Yang, S. Y. Cheng, Y. W. Chen-Yang, Preparation and properties of polyimide/silica hybrid nanocomposites, Polym. Compos. 128 (2007) 575-581.

[4] P Santhana Gopala Krishnan, Andy Eko Wisanto, Stephen Osiyemi, Chen Ling, Synthesis and properties of BCDA-based polyimide-clay nanocomposites, Polym. Int. 56 (2007) 787-795.

[5] T. Agag, T. Koga, T. Takeichi, Studies on thermal and mechanical properties of polyimide-clay nanocomposites, Polym. 42 (2001) 3399-3408

[6] Byoung Wook Jo, Kyung Hyum Ahn, Seung Jong Lee, Effect of thermal history during drying and curing process on the chain orientation of rod-shaped polyimide, polym. 55 (2014) $5829-5836$.

[7] Gayathri R. Sharma, Cora Lind, Maria R. Coleman, Preparation and properties of polyimide nanocomposites with negative thermal expansion nanoparticle filler, Mater. Chem. Phys. 137 (2012) 448-457.

[8] Juan Yang, Yongsen Yang, Qinqin Liu, Guifang Xu, Xiaonong Cheng, Preparation of negative thermal expansion $\mathrm{ZrW}_{2} \mathrm{O}_{8}$ powder and its application in polyimide/ZrW2O8 composites, J. Mater. Sci. Technol. 26 (2010) 665-668. 
[9] HongChao Wu, Mark Rogalski, Michael R. Kessler, Zirconium Tungstate/Epoxy Nanocomposites: Effect of Nanoparticle Morphology and Negative Thermal Expansivity. ACS Appl. Mater. Interfaces 5 (2013): 9478-9487.

[10] Prashanth Badrinarayanan, Mark Rogalski, Hong Chao Wu, Xiaofeng Wang, Wonjong Yu, Michael R. Kessler, Epoxy Composites Reinforced with Negative-CTE $\mathrm{ZrW}_{2} \mathrm{O}_{8}$ Nanoparticles for Electrical Applications. Macromol. Mater. Eng. 298 (2013): 136-144.

[11] Xinxin Chu, Rongjin Huang, Huihui Yang, Zhixiong Wu, Junfeng Lu, Yuan Zhou, Laifeng Li, The cryogenic thermal expansion and mechanical properties of plasma modified $\mathrm{ZrW}_{2} \mathrm{O}_{8}$ reinforced epoxy, Mater. Sci. Eng. A 528 (2011) 3367-3374.

[12] Zhenping Shang, Changli Lü, Xiaodan Lü, Lianxun Gao, Synthesis and properties of silica-polyimide hybrid films derived from colloidal silica particles and polyamic acid, J. Appl. Polym. Sci. 109 (2008) 3477-3483.

[13] Jun-Wei Zha, Zhi-Min Dang, Tao Zhou, Hong-Tao Song, George Chen, Electrical properties of $\mathrm{TiO}_{2}$-filled polyimide nanocomposite films prepared via an in situ polymerization process, Synth. Met. 160 (2010) 2670-2674.

[14] Zhu-Mei Liang, Jie Yin, Hong-Jie Xu, Polyimide/montmorillonite nanocomposites based on thermally stable, rigid-rod aromatic amine modifiers, Polym. 44 (2003) 1391-1399.

[15] Wan-Jung Chou, Cheng-Chien Wang, Chuh-Yung Chen, Characteristics of polyimide-based nanocomposites containing plasma-modified multi-walled carbon nanotubes, Compos. Sci. Technol. 68 (2008) 2208-2213.

[16] Pei-Chun Chiang, Wha-Tzong Whang, Mei-Hui Tsai, Sheng-Chang Wu, Physical and mechanical properties of polyimide/titania hybrid films, Thin Solid Films. 447-448 (2004) 
359-364.

[17] Juntao Wu, Shiyong Yang, Shengqiang Gao, Aijun Hu, Jingang Liu, Lin Fan, Preparation, morphology and properties of nano-sized $\mathrm{Al}_{2} \mathrm{O}_{3}$ /polyimide hybrid films, Eur Polym. J. 41 (2005) 73-81.

[18] Mehmet Cetinkol, Angus P. Wilkinson, Pressure dependence of negative thermal expansion in $\mathrm{Zr}_{2}\left(\mathrm{WO}_{4}\right)\left(\mathrm{PO}_{4}\right)_{2}$, Solid State Commun. 149 (2009) 421-424.

[19] J.S.O. Evans, T.A. Mary, A.W. Sleight, Negative Thermal Expansion in a large Molybdate and Tungstate Family, J. Solid State Chem. 133 (1997) 580-583.

[20] T.A. Mary, A.W. Sleight, Bulk thermal expansion for tungstate and molybdates of the type $\mathrm{A}_{2} \mathrm{M}_{3} \mathrm{O}_{12}$, J. Mater. Res. 14 (1999) 912-915.

[21] J.S.O. Evans, T.A. Mary, A.W. Sleight, Structure of $\mathrm{Zr}\left(\mathrm{WO}_{2}\right)\left(\mathrm{PO}_{4}\right)_{2}$ from powder X-Ray Data: Cation ordering with no superstructure, J. Solid State Chem. 120 (1995) 101-104.

[22] Xian-Sheng Liu, Fu-Xing Cheng, Jun-Qiao Wang, Wen-Bo Song, Bao-He Yuan, Er-Jun Liang, The control of thermal expansion and impedance of $\mathrm{Al}-\mathrm{Zr}_{2}\left(\mathrm{WO}_{4}\right)\left(\mathrm{PO}_{4}\right)_{2}$ nano-cermets for near-zero-strain Al alloy and fine electrical components, J. Alloys Compd. 553 (2013) 1-7.

[23] J.Z. Tao, A.W. Sleight, The role of rigid unit modes in negative thermal expansion, J. Solid State Chem. 173 (2003) 442-448.

[24] Cora Lind, Two Decades of Negative Thermal Expansion Research: Where Do We Stand? Materials. 5 (2012) 1125-1154.

[25] Mehmet Cetinkol, Angus P. Wilkinson, Cora Lind, In situ high-pressure synchrotron X-ray diffraction study of $\mathrm{Zr}_{2}\left(\mathrm{WO}_{4}\right)\left(\mathrm{PO}_{4}\right)_{2}$ up to 16GPa, Phys. Rev. B 79 (2009) 224118:1-224118:10.

[26] Toshihiro Isobe, Takuya Umezome, Yoshikazu Kameshima, akira Nakajima, Kiyoshi Okada, 
Preparation and properties of negative thermal expansion $\mathrm{Zr}_{2} \mathrm{WP}_{2} \mathrm{O}_{12}$ ceramics, MATER RES BULL. 44 (2009) 2045-2049.

[27] Qin Jiaqiang, Li Zhen, Gu Yi, Study on Morphology Control and How to Affect mechanical Properties of Polyimide/Silica Hybrid Films, J. Appl. Polym. Sci. 118 (2010) 2772-2777.

[28] Xinwei Shi, Hong Lian, Ruiqiong Qi, Libin Cui, Ning Yao, Preparation and properties of negative thermal expansion $\mathrm{Zr}_{2} \mathrm{WP}_{2} \mathrm{O}_{12}$ powders and $\mathrm{Zr}_{2} \mathrm{WP}_{2} \mathrm{O}_{12} /$ TiNi composites, MATER SCI ENG B-ADV. 203 (2016) 1-6.

[29] Yurong Shi, Yuhua Wang, Dan wang, Bitao Liu, Yanhun Li, Lan Wei, Synthesis of hexagonal prism $(\mathrm{La}, \mathrm{Ce}, \mathrm{Tb}) \mathrm{PO}_{4}$ phosphors by precipitation method, Cryst. Growth Des. 12 (2012) $1785-1791$.

[30] Xiujuan Sun, Juan Yang, Qinqin Liu, Xiaonong Cheng, Influence of sodium dodecyl benzene sulfonate (SDBS) on the morphology and negative thermal expansion property of $\mathrm{ZrW}_{2} \mathrm{O}_{8}$ powders synthesized by hydrothermal method, J. Alloys Compd. 481 (2009) 668-672.

[31] Vinila Bedekar, O. D. Jayakumar, J. Manjanna, A. K. Tyagi, Synthesis and magnetic studies of nano-crystalline $\mathrm{GdFeO}_{3}$, Mater. Lett. 62 (2008) 3793-3795.

[32] Cora Lind, Maria R. Coleman, Leah C. Kozy, Gayathri R. Sharma, Zriconium tungstate/polymer nanocomposites: Challenges and opportunities, Phys. Status Solidi B. 248 (2011) 123-129

[33] Gayathri R. Sharma, Polyimide Nanocomposites Based on Cubic Zirconium Tungstate [D], Toledo, the University of Toledo, 2009.

[34] Minoo Naebe, Jing Wang, Yuhua Xue, Xungai Wang, Tong Lin, Carbon Nanotube Reinforced Rigid-Rod Polyimide, J. Appl. Polym. Sci. 118 (2010) 359-365.

[35] Wei-Dong Liu, Bao-Ku Zhu, Jian Zhang, You-Yi Xu, Preparation and dielectric properties of 
polyimide/silica nanocomposite films prepared from sol-gel and blending process, Polym. Adv. Technol. 18 (2007) 522-528.

[36] Ding Mengxian, polyimides synthesized by polyamic acid, in: Polyimides-Chemistry, Relationship between Structure and Properties and Materials, Science Press, Beijing , 2006, pp. 75-76.

[37] Giuseppe Ragosta, Pellegrino Musto, Mario Abbate, Gennaro Scarinzi, Compatibilizing Polyimide/Silica Hybrids by Alkoxisilane-Terminated Oligoimides: Morphology-Properties Relationships, J. Appl. Polym. Sci. 121 (2011) 2168-2186.

[38] FOIL A. MILLER and CHARLES H. WILKINS, Infrared Spectra and Characteristic Frequencies of Inorganic Ions: Their Use in Qualitative Analysis, ANALYTICAL CHEMISTRY. 24 (1952) $1253-1294$.

[39] Cs.Balázsi, M.Farkas-jahnke, I. Kotsis, L. Petrás, J. Pfeifer, The observation of cubic tungsten trioxide at high-temperature dehydration of tungstic acid hydrate, Solid State Ionics 141-142 (2001) 411- 416.

[40] Z. Ahmad, J. E. Mark, Polyimide-Ceramic Hybrid Composites by the Sol-Gel Route, Chem. Mater. 13 (2001) 3320-3330.

[41] Mahmoud A. Hassan, Mousa M. Abdul-Gader Jafar, Frequency dependence of loss tangent of thermally annealed undoped lead iodide crystals in the dark, Nucl. Instrum. Methods Phys. Res., Sect. A 566 (2006) 526-535.

[42] Yue Guan, Daming Wang, Guangliang Song, Guodong Dang, Chunhai, Chen, Hongwei Zhou, Xiaogang Zhao, Novel soluble polyimides derived from 2, 2'-bis[4-(5-amino-2-pyridinoxy)phenyl] hexafluoropropane: preparation, characterization, and optical, dielectric properties, Polym. 55 
(2014) 3634-3641.

[43] Hung T. Vo, Frank G. Shi, Towards model-based engineering of optoelectronic packaging material: dielectric constant modeling, Microelectron. J. 33 (2002) 409- 415.

[44] T. Badapanda, V. Senthil, S. Anwar, L. S. Cavalcante, N. C. Batista, E. Longo, Structure and dielectric properties of polyvinyl alcohol/barium zirconium titanate polymer-ceramic composite, CURR APPL PHYS. 13 (2013) 1490-1495.

[45] Zhi-Min Dang, You-Qin Lin, Hai-Ping Xu, Chang-yong Shi, Sheng-Tao Li, Jin-Bo Bai, Fabrication and dielectric characterization of advanced $\mathrm{BaTiO}_{3} /$ Polyimide nanocomposite films with high thermal stability, Adv. Funct. Mater. 18 (2008) 1509-1517. 
With increasing of ZWP in the composites, the CTEs of the ZWP/PI were reduced. A $40 \mathrm{wt} \%\left(19.6 \mathrm{vol} \%\right.$ ) ZWP loading gives a $32.5 \%$ (about $15 \times 10^{-6} / \mathrm{K}$ ) reduction of CTE of the composite.

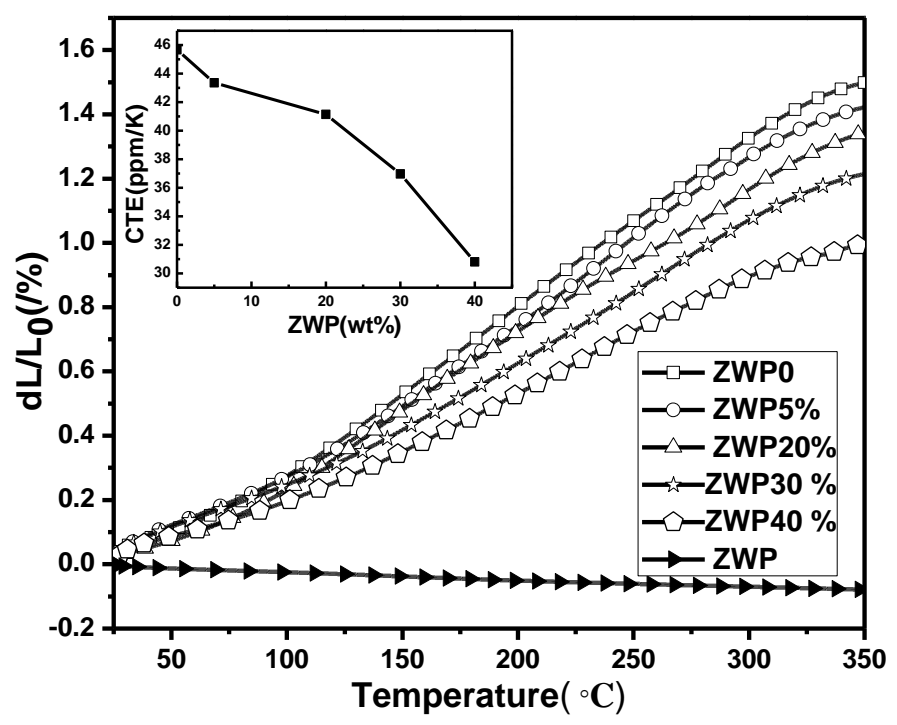

